\title{
Vorstellung der Kandidaten für die Präsidiumswahl
}

Werner Ballmann. Ich wurde am 11. April 1951 in Hillesheim (Eifel) geboren. Die Volksschule besuchte ich im Nachbarort Oberbettingen, das Abitur machte ich 1970 am St. Matthias-Gymnasium in Gerolstein. Nach Wehr- und Ersatzdienst studierte ich ab 1971 Mathematik in Bonn. Das Diplom in Mathematik erhielt ich 1976, promoviert wurde ich 1979. Danch arbeitete ich als Assistent am Mathematischen Institut der Universität Bonn. Im akademischen Jahr 198081 erhielt ich ein Forschungsstipendium der DFG und besuchte die University of Pennsylvania in Philadelphia. Ab Herbst 1984 war ich Visiting Associate Professor an der University of Maryland, von Herbst 1985 an Associate Professor. Im Sommer 1986 wurde ich als außerordentlicher Professor Nachfolger von Ernst Ruh an der Universität Bonn, im Herbst 1987 folgte ich einem Ruf auf ein Ordinariat an der Universität Zürich. Seit Oktober 1989 bin ich als Nachfolger von Wilhelm Klingenberg ordentlicher Professor an der Universität Bonn. Ich bin verheiratet und habe zwei Töchter.

Mein Arbeitsgebiet ist die Differentialgeometrie, die ich in Bonn bei Wilhelm Klingenberg, Hermann Karcher und Ernst Heintze kennenlernte. Geprägt haben

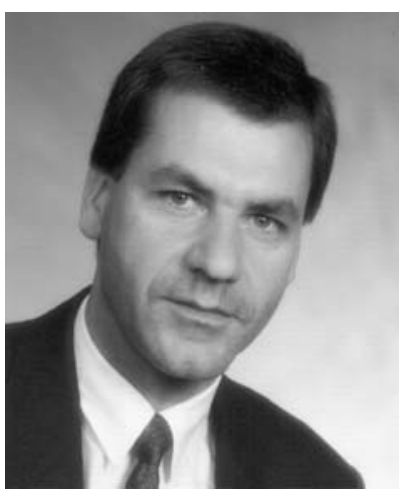

Jürg Kramer. Geboren am 3. Juni 1956 in Zürich. 1975 Matura. Studium der Mathematik, Physik und Astronomie an der Universität Basel. 1980 Diplom in Mathematik an der Universität Basel. 1985 Promotion zum Dr. phil. an der Universität Basel. 1992 Habi-

litation in Mathematik an der ETH Zürich.

Berufliche Tätigkeiten: 1980-85 Assistent an der Universität Basel. 1986 Postdoctoral Fellow an der Harvard University. 1987 Research Fellow am Mathematical Sciences Research Institute in Berkeley. 1987-88 wissenschaftlicher Mitarbeiter an der Gesamthochschule/Universität Wuppertal. 1989-92 Oberassistent an der ETH Zürich. 1993 Professor an der Université Laval (Québec, Canada). Seit 1994 Professor für Mathematik an der HumboldtUniversität zu Berlin.

Arbeitsgebiete: Arithmetische algebraische Geome- mich auch Vorlesungen von Stefan Hildebrandt zur Variationsrechnung, Friedrich Hirzebruch zu charakteristischen Klassen und Jacques Tits zur algebraischen Topologie. Innerhalb der Differentialgeometrie habe ich mich insbesondere mit geschlossenen Geodätischen, geodätischen Flüssen, symmetrischen und singulären Räumen beschäftigt. Seit einigen Jahren arbeite ich auch über die Spektraltheorie geometrischer Operatoren. Meine Arbeit hat mannigfaltige Beziehungen zu algebraischer Topologie, geometrischer Gruppentheorie, dynamischen Systemen und stochastischen Prozessen.

Als Student habe ich in der Bonner Fachschaft Mathematik/Physik mitgearbeitet. Die vielen unergiebigen Sitzungen mit Kommilitonen, die den politischen Jargon der Zeit perfekt beherrschten und in langen Reden nichts sagten, haben meine Einstellung zur Gremienarbeit geprägt. Wichtig scheint mir, dass wir uns von der Politik und den vielen, häufig überflüssigen Verwaltungsaufgaben nicht erdrücken lassen. Es muss Zeit bleiben für unsere eigentlichen Aufgaben, Forschung und Lehre. Die DMV sollte versuchen dazu beizutragen, dass uns die nötige Muße dafür bleibt.

trie (Arakelov-Geometrie) und die vielfältigen Beziehungen zur Theorie der automorphen Formen (Modulformen); Theorie der ganzzahligen quadratischen Formen und lineare Abhängigkeiten von Thetareihen. Fachwissenschaftliche und -didaktische Fragestellungen zur Lehramtsausbildung; Förderung von mathematischen Begabungen (Berliner Netzwerk mathematisch-naturwissenschaftlich profilierter Gymnasien); Popularisierung von Mathematik.

Zur Arbeit in der DMV: Auf Anfrage der Findungskommission habe ich mich gerne zur Kandidatur für das Amt des Schatzmeisters der DMV zur Verfügung gestellt. Durch meine Tätigkeit als Geschäftsführender Direktor des Instituts für Mathematik der Humboldt-Universität während der Jahre 1998 bis 2002 konnte ich wertvolle Erfahrungen in der Verwaltung von Geldern erwerben, welche bei der Ausübung des Schatzmeisteramtes von Nutzen sein werden. Über die Schatzmeister-Tätigkeit hinaus möchte ich auch meine Erfahrungen zur Mathematikausbildung an der Schnittstelle zwischen Schule und Universität in die DMV einbringen, um damit beispielsweise einen Beitrag zu den Reformbemühungen der DMV bei der Lehramtsausbildung zu leisten. 


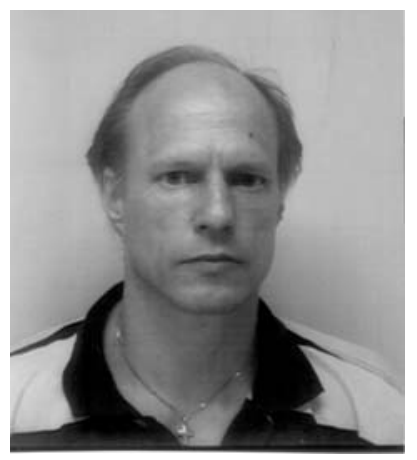

Michael Röckner. Geboren am 15. Februar 1956 in Herford, verheiratet, 3 Kinder. Hobbys (außer der Mathematik): Sport (insbesondere Fußballspielen); Modelleisenbahnen. 1976-1982 Studium der Mathematik und Physik in Bielefeld. 1984 Promotion und 1987 Habilitation an der Universität Bielefeld. 1984-1985 Gast von Professor Dynkin an der Cornell University, 1986-1990 zunächst Lecturer, dann Reader an der University of Edinburgh. 19901994 Professor an der Universität Bonn, ab 1994 Professor an der Universität Bielefeld. Forschung: Meine Forschungsinteressen liegen hauptsächlich im Grenzbereich zwischen Analysis und Wahrscheinlichkeitstheorie (,Stochastische Analysis") mit starker Orientierung an Anwendungen in der mathematischen Physik. Zur Zeit beschäftige ich mich hauptsächlich mit stochastischen partiellen Differentialgleichungen und unendlichen interagierenden Teilchensystemen. Lehre: Ich hatte bislang 20 Diplomandinnen und Diplomanden und fünf Promovenden, die unter meiner Anleitung ihre Arbeiten geschrieben und abgeschlossen haben.

Seit 1997 bin ich Dekan bzw. Prodekan der Fakultät für Mathematik in Bielefeld. Ich habe dabei die Zei- ten des „Qualitätspakts“ in NRW „,durchlitten“ und währendessen viel Energie in den Erhalt von Mathematikstellen investiert. Spätestens im Oktober 2002 werde ich das Amt des Dekans abgeben und würde dann, wenn ich das Vertrauen der Wähler erhalte, gern im Präsidium der DMV mitarbeiten.

Ich hoffe, dort meine seit 1997 gesammelte politische Erfahrung im Sinne unserer Wissenschaft einbringen zu können. Dabei liegt mir vor allem der mathematische Nachwuchs am Herzen. Bei (meiner Ansicht nach) immer schlechter werdenden Rahmenbedingungen für junge Wissenschaftlerinnen und Wissenschaftler allgemein, gehen insbesondere in Fächern wie der Mathematik mit sehr guten Berufsaussichten für ihre Absolventen außerhalb der Universität (oder im Ausland) zu viele begabte und passionierte junge Menschen der Wissenschaft (in Deutschland) verloren. Ein weiterer Bereich meines besonderen Interesses sind neue Studienstrukturen. Sollten, so wie es zur Zeit unvermeidlich erscheint, gestufte Abschlüsse (wie der BSc nach sechs Semestern und dann der MSc nach zehn Semestern) eingeführt werden, so sehe ich hier eine Möglichkeit, die Ausbildung der Studierenden $\mathrm{zu}$ verbessern und mit dem MSc insbesondere wegen der dann wieder 10-semestrigen Studienzeit das Niveau des Diploms auf jeden Fall zu halten oder sogar noch anzuheben. Natürlich gibt es in beiden angesprochenen Bereichen bereits Initiativen, an denen die DMV beteiligt ist. Ich würde mich freuen, als Präsidiumsmitglied der DMV hier meinen Beitrag leisten zu können.

\section{Einladung zur Mitgliederversammlung der DMV}

Im Rahmen der der DMV-Tagung in Halle findet am 19. 9. 2002 um 17.30 Uhr im Audimax (Universitätsplatz) eine Mitgliederversammlung statt. Ich möchte Sie hiermit im Namen des Präsidenten herzlich dazu einladen.

\section{Vorschlag für die Tagesordnung}

Top 0: Genehmigung der Tagesordnung

Top 1: Bericht des Präsidenten

Top 2: Vorstellung der neuen Präsidiumsmitglieder

Top 3: Bericht des Schatzmeisters und der Kassenprüfer

Top 4: Entlastung der Vereinsorgane
Top 5: Wahl der Kassenprüfer

Top 6: Mitgliederbeiträge

Top 7: Diskussionspunkt: „Wie kann die DMV nach innen und nach außen attraktiver werden?"

Top 8: Verschiedenes gez. Behrends (Schriftführer) 